\title{
Requirements for Standards and Regulations in AI-Enabled Prognostics and Health Management
}

\author{
Sunday Ochella ${ }^{1, \mathrm{a}}$, Mahmood Shafiee ${ }^{2, \mathrm{~b}}$, Chris Sansom ${ }^{1, \mathrm{c}}$ \\ ${ }^{1}$ Department of Energy and Power, Cranfield University, College Road, Befordshire MK43 0AL, UK \\ ${ }^{2}$ Mechanical Engineering Group, School of Engineering, University of Kent, Canterbury CT2 7NT, UK \\ a Sunday.Ochella@cranfield.ac.uk; \\ ${ }^{\mathrm{b}} \mathrm{m}$. shafiee@kent.ac.uk; \\ cc.l.sansom@cranfield.ac.uk \\ ${ }^{\mathrm{b}}$ Corresponding Author
}

\begin{abstract}
The fundamental understanding of the core aspects of prognostics and health management (PHM) as a field of practice is somewhat fully established. However, the various approaches used in the field have continuously evolved. With the recent surge in the adoption of artificial intelligence (AI) algorithms for predictive analytics, datadriven PHM is now more prominent. Notwithstanding the popularity of AI approaches, actual adoption and implementation in fielded systems has been minimal. One of the reasons for this is the lag in an ancillary area, which is the development of corresponding standards and regulations to guide the practice. This paper aims to synthesize various studies in the literature regarding standards and regulations in data-driven PHM and then sets out the necessary requirements for a standards and regulations regime to support the full adoption of AI-enabled PHM. An acceptability criterion is proposed, which incorporates the various factors that must be considered for verification, validation, and certification of AI-enabled PHM technologies. The use of the acceptability criterion is demonstrated, which will potentially be very useful to certification bodies and regulatory agencies in the process of approving AI-enabled PHM for use in safety-critical assets.
\end{abstract}

Keywords-artificial intelligence (AI); prognostics and health management (PHM); standards and regulations; datadriven prognostics; remaining useful life (RUL).

\section{INTRODUCTION}

Prognostics and health management (PHM) involves the key tasks of diagnostics, prognostics, and decisionsupport, each of which can be further sub-divided, as will be shown later in Section II of this paper. Of the three key tasks, diagnostics is an area that is well established, along with the decision support derived therefrom. Prognostics, on the other hand, is still an evolving area due to the inherent difficulty of making predictions. A major endeavor in prognostics is the prediction of the remaining useful life (RUL) of an asset. Approaches used for RUL prediction include model-based methods which use the physics of failure for the physical system as a basis, datadriven methods which use operations, inspections and sensor data from the system, or hybrid/fusion approaches which combine both physics-based and data-driven methods [1]. In recent time, the increased complexity of physical systems means that it is impossible to model them using a simple physics-of-failure approach. Fortunately, advances in sensor technology mean that lots of data can be gathered from such systems, and when combined with now readily available high computing power and artificial

S. Ochella thanks the Petroleum Technology Development Fund in Nigeria for sponsoring his $\mathrm{PhD}$ research. intelligence (AI) algorithms, meaningful insights can be gained.

Since the diagnostics aspect of PHM is well established, most of the existing standards guiding the practice of PHM have been adapted from diagnostics applications. Some of these standards have been reviewed in detail in different papers [2]-[6]. Some of the referenced papers contain quite detailed treatises on standards issued by the International Organization for Standardization (ISO), the Society of Automotive Engineers (SAE), the Institution of Electrical and Electronics Engineers (IEEE) and the Machinery Information Management Open System Alliance (MIMOSA), which will not be repeated in this paper. However, all the mentioned standards tend to be agnostic to the approach used. In this regard, this work will highlight standards specific to the use of AI algorithms, and to datadriven prognostics, in general. Although some of the proposals presented in this paper may be applied directly to AI-enabled diagnostics, not much attention is paid to diagnostics as it has been covered by existing standards.

Practically all the existing versions of AI algorithms have been used for prognostics [1]. The algorithms are rapidly unraveling, and so are their applications for prognostics. Consequently, regulating the use of AIenabled prognostics for fielded systems must incorporate enough flexibility to adapt to the rapidly evolving advancements in the field. Regulations, while ensuring safety, must at the same time not pose insurmountable bottlenecks or stifle growth. The development of standards and regulations for a particular technology inherently lags the technology itself. However, beyond proof of concept and actual deployment of some test facilities, standards and regulations should typically converge with advances in the technology. The focus of the discourse around regulating AI has been on ethical, legal and data privacy issues, and this is reflected in the national strategies for AI which are being adopted by different countries in Europe [7]. With regards to prognostics, this paper proposes a semiquantitative approach to verification and validation, that draws of practices form safety and reliability engineering. The outcome of such an approach can then be used as a basis for certification of the PHM technology and serve as baseline for regulatory monitoring and compliance.

The remaining part of this paper is structured as follows. Section II provides a brief update of extant standards and regulations that intersect with some aspects of the use of AI algorithms. Section III presents an analysis of the various factors that feed into the decision to 
ultimately adopt any AI-enabled PHM solution, culminating in the proposal of an acceptability criterion. Section IV considers the hardware and software issues to enable seamless application of AI-enabled PHM solutions to legacy assets from a standards and regulations perspective and with a view towards life extension for such legacy facilities. Section V concludes the paper.

\section{EXTANT STANDARDS AND REGULATIONS}

Engineering practice is typically guided by standards, while the products of engineering endeavors are regulated by government statutes and regulations. Standards embody guidelines, approaches and concepts and must not be mistaken for strict procedures [8]. Nonetheless, using standards as guides help with the process of verification and certification, and is therefore crucial to the process of regulatory compliance. Some other obvious reasons for standardization include minimizing repeated designs of similar systems, enhancing compatibility and interoperability [9], harmonizing the lexicon of professional practice in a particular field [10] and ensuring that best practices are maintained, across board, within the profession. Furthermore, standards and regulations help to increase trustworthiness, and hence adoption of technology. The intersections between standardization and the need for regulations, in the context of AI-enabled PHM, are the aspects of trustworthiness, safety, and legal liability in case of failure. These factors, amongst others, will form the bedrock of the AI-enabled PHM acceptability criterion proposed in this paper.

\section{A. Standards}

This section briefly discusses the existing standards that overlap with AI-enabled PHM, especially as regards data management and cross-platform compatibility in terms of information exchange. To provide a uniform platform for design, development, and deployment of PHM technologies, the need for uniform terminologies was identified early. A good attempt at defining the boundaries and establishing uniformity in PHM lexicon was put forth in reference [10]. Further, issues around data formats, ease of interconnection, integration and other cross-platform issues were addressed in MIMOSA OSA-CBM [11], MIMOSA OSA-EAI [12], the ISO-13374 series and the IEEE Std 1232 Artificial Intelligence Exchange and

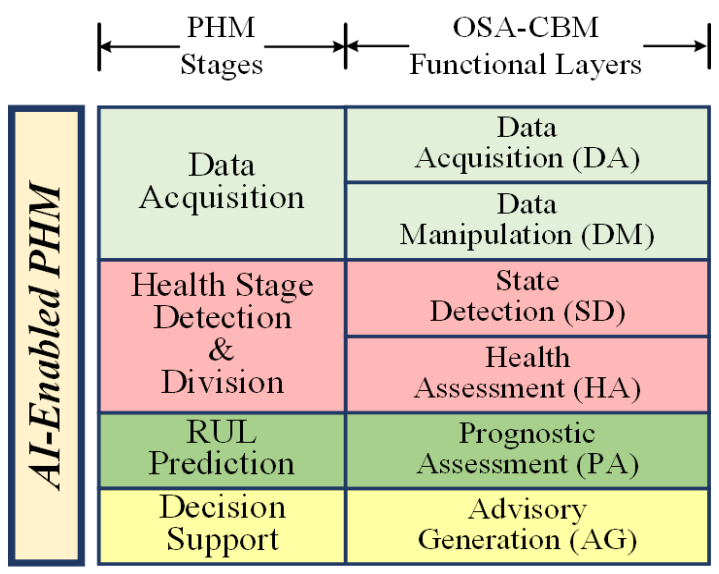

Figure 1. Various stages and subdivisions of AI-enabled PHM
Service Tie to All Test Environments (AI-ESTATE). In general, AI-enabled PHM involves four stages: data acquisition and processing, health stage detection and division, RUL prediction, and maintenance decisionmaking [13]. Fig. 1 shows how the OSA-CBM maps to each of these four stages while Table I provides a list of some extant standards and how they map to the layers of AI-enabled PHM within the OSA-CBM architecture.

TABLE I. STANDARDS FOR DIFFERENT STAGES IN AI-BASED PHM

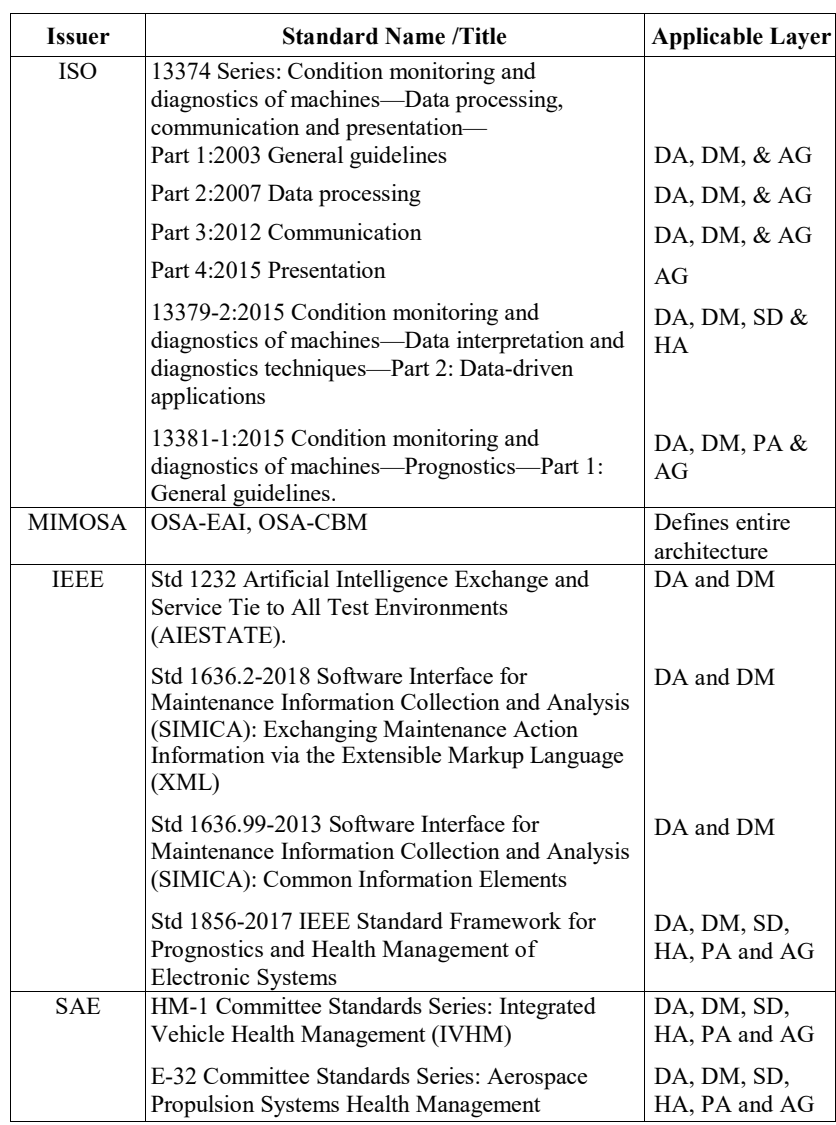

An important observation from Table I is that the listed standards mostly apply to data acquisition, data processing, and advisory generation or information presentation to help with decision-support. Other existing standards are United States Military Handbooks, which address similar areas like those addressed by the ISO (with some in collaboration with the International Electrotechnical Commission, IEC) and the SAE [14]. Other standards, not cited here, but duly discussed in [2]-[6], as stated earlier, dwell on the various stages of conventional PHM process, but not particularly on AI-based methods.

\section{B. Regulations}

Typically, for any technology to be approved for use, vital areas of concern to governments and regulatory agencies include safety, security, benefits and costs, public trust, and ethical concerns, all of which must be addressed through a comprehensive risk assessment and management plan. As such, regulatory agencies over time, vest the responsibility for demonstrating safety of facilities on asset managers. Standard risk assessment and management procedures can be developed to critically assess AIenabled PHM systems, which may be modelled in a similar 
fashion to the ISO/IEC/IEEE International Standard 160852020 for systems and software engineering life cycle processes [15]. Fundamentally, a regulations regime for AIenabled PHM must address the following areas.

a) Safety - an approach similar to safety case development can be extended to the use of AI in PHM systems. For this to be effective, the areas of explainability and interpretability of AI must be adequately addressed. At a minimum, AI-enabled PHM solutions must attain or beat the level of safety and reliability achievable by conventional systems, usually assigned as Safety Integrity Levels (SIL). As early as 2001, the UK's Health and Safety Executive (HSE) identified the need for safety in industrial use of artificial neural networks [16]. The HSE report highlighted that, from a safety perspective, there is the need to minimize over-complexity of models (thus aiding explainability), and for predictions to be interpretable. Furthermore, since data for high consequence, low probability scenarios are scarce, it should be required that the optimization process for AI-based algorithms heavily penalize erroneous predictions around such regions, since they are mostly safety-critical [17]. To be meaningful and therefore increase confidence, predictions must also necessarily incorporate uncertainty quantification.

b) Cyber-security - the interconnectedness achieved by cyber-physical systems, of which AI-enabled PHM systems are a part, implicitly introduces cyber-security challenges. So, from a safety, security and legitimacy standpoint, overall cyber-security issues must be adequately addressed before any credit can be taken for the validity of predictions. Data security must be foolproof, since prognostics results ultimately depend on the legitimacy of the data used for training and updating of predictive models.

c) Costs and benefits - at the core of deploying new technologies in fielded systems is the demonstration of overriding costs and benefits, when compared to existing systems. This may appear to be a major concern for only the asset owners. However, all government directives or regulations can indeed render innovation unviable because compliance to such regulations can potentially raise costs disproportionately. Regulations must therefore be drawn up to not only address safety, but also ensure that the costbenefit implications are duly assessed.

d) Flexibility - AI is still evolving, and regulations must be flexible enough to adapt to rapid changes in development in the technologies deploying AI, like PHM systems. Governments across the world have recognized the huge potentials of AI in relation to the Industrial Internet of Things (IIoT) and smart manufacturing, and the attempt to regulate AI must be carefully measured so that innovation is not inadvertently stifled. A workable proposal around this is the use of regulatory sandboxes to allow for the mutual growth of both AI-enabled technologies and the corresponding regulations.

e) Ethical perspective - due to the fact that major concerns are usually about public-facing AI products, most of the approaches towards the regulation of AI-enabled technologies have so far been from an ethical perspective.
In relation to AI-enabled PHM, there is an intersection as regards automated decision-making technologies, which has led to attempts by professional societies like the IEEE to address these concerns by drafting the Ethically Aligned Design Standard IEEE P7000 [18]. Again, the recurring points about explainability and interpretability can help improve transparency and allay any concerns regarding the ethical aspects of AI-based PHM systems.

f) Legal perspective - legal frameworks need to be set up to determine culpability and compensation issues that may arise from accidents due to failures attributable to AI-enabled PHM systems. The European Union (EU) has updated its Product Liability Directive to account for IIoT and intelligent autonomous systems [19]. Also, the regulatory implications for the safety of AI-based modules in original equipment manufacturer (OEM) packages or machinery are now being addressed. For example, the EU Machinery Directive is now updated to address IIoT issues [20]. There is also the need for predictions and performance logging and recording, to help during audits and root cause analysis as parts of incident investigations.

g) Trustworthiness - for an AI-enabled PHM system to be trustworthy, it must have a clearly defined purpose; be legitimate in terms of data quality, governance and risk management; be able to verifiably perform its intended functions; provide decision-support capabilities that ensure increased human-machine interdependence; and have a transparent impact on stakeholders [21]. Different approaches to achieve trustworthiness of AI systems by demonstrating safety, security, reliability, resiliency, and availability are specified in ISO/IEC TR 24028:2020 [22].

All the key areas discussed in (a)-(g) will underpin the verification, validation, assurance, and certification that should form the core of a regulations regime for AI-enabled PHM systems. In addition, post-deployment runtime monitoring and regulation enforcement should be similar to subsisting requirements for reporting and compliance.

\section{Best Practices}

Similar to the process of developing new technology and qualifying it for use, a strict process of technology qualification needs to be followed. The technology qualification process is well established for conventional systems, using well-known reliability methods to ensure that all failure modes and physics of failures are addressed. Also, software engineering practices such as audit trails, workflows, bias testing, verification and validation testing and explainable user interfaces are well established [23]. An amalgam of both practices, streamlined for AI-based workflows, can be adopted for AI-enabled PHM. Furthermore, safety engineering practices which help explore physical systems and the understanding of how they fail can be employed as an additional layer of check to guide decision-making [24]. Such relevant tools may include fault tree analysis (FTA), failure modes, effects and (criticality) analysis (FME(C)A), or Event Trees (Attack Trees in cybersecurity).

Training an AI-enabled PHM model on specific training data introduces bias which must be offset through sensitivity analysis, uncertainty quantification and testing on out-of-sample data to ascertain true performance. This 
must be a minimum requirement for assurance and eventual certification. In addition, the various plans on how to integrate AI-based prognostics systems into asset, data and organizational management structures must be vetted and assured, preferably by independent third-party to eliminate potential familiarity bias by in-house engineers. DNVGLRP-0510 provides a framework for assurance of datadriven algorithms and models [25].

From a regulatory standpoint, it must be further emphasized that independent third-party testing, verification, and validation remain vital. For AI-based systems, verification should probe the key concerns of repeatability, explainability and interpretability. Methods for explaining AI-based predictions include the use of counterfactuals or post hoc (retrospective) methods, causal methods incorporating expert knowledge, and the use of interactive/exploratory user interfaces [23], [26]. On the basis of independent third-party verification, AI-based PHM systems can then be certified in compliance with subsisting regulatory requirements, as is typically the practice. Post-certification, and after deployment in fielded systems, continuous monitoring and feedback is important. Conventional ways of maintaining the overall safety culture in organizations through personnel training, competency development, detailed failure reporting and incident investigations must be adhered to.

\section{FULFILLING REGULATORY COMPLIANCE}

\section{A. Further Requirements}

In addition to the previously discussed areas which should be considered for an effective regulation regime, this section sets out basic requirements for safety-critical assets, culminating in the proposal of a flexible, robust, and user-definable acceptability criterion for AI-enabled PHM. Safety-critical assets or systems are those whose failure can lead to serious injury, loss of life or significant economic consequences. Some critical infrastructure where AI-based PHM are being deployed include electric power systems, oil and gas generation and distribution, water supply systems, road, rail and air transportation systems [27]. Clearly, most of these systems are public-facing and must be regulated to ensure public as well as industrial safety. To attain high confidence in the decision support derived from AI-enabled PHM for such critical infrastructure, the following considerations should be made.

1) Besides standards and regulations, policies are another important layer in the overall drive towards effective PHM implementation. While standards are driven at the level of professional or standardization organizations and regulations are driven at the level of the government, policies are driven at the level of the organization or asset operator [28]. For each of the important factors highlighted in this paper, organizational policies should be updated or formulated to address successful implementation and continuous monitoring of AI-enabled PHM systems. E.g., data governance policy, cyber-security policy, safety policy, legal and ethical policy, etc.

2) Since data for high consequence and low probability events are typically scarce, AI algorithms should be adapted to such tail events by generating data around tail events based on causal knowledge of the physical system, thus enabling the infusion of some learning data points within the low-probability region [29]. For such scenarios, moreover, constraints can be imposed on predictions from the AI algorithm so as to lie within known limits of operations of such physical systems.

3) Fail-safe operations should be derived by exploiting ensemble learning such that, in the scenario that there is no consensus from the multiple predictors within the ensemble, intelligent agents may make decisions regarding the optimal prediction while also prompting human agents for decision-making [27].

4) There should be a clear delineation of the conditions or assumptions under which prognostics were made and the boundaries of validity must accompany any predictions.

5) Concepts of explainable AI (XAI) should be incorporated, with the provision of interactive and exploratory user interfaces that ensure that the user understands the accuracy of predictions and can interpret them using the associated uncertainty bounds. The user should also understand when the failure will occur, what the likely failure mode will be and when to take proactive action to avert failure.

\section{B. Acceptability Criterion}

In this section, all the critical factors for the effective implementation of a regulatory regime are harnessed and consolidated to propose a unifying criterion for accepting an AI-enabled PHM system or module. For consideration during certification or as part of the regulatory approval process, all the important factors mentioned should be checked off as either satisfactory or unsatisfactory. If the results from such a process are collated as an array, $F$, we propose an acceptability criterion, $A_{c}$, as given in (1):

$$
A_{c}=\boldsymbol{\beta} \boldsymbol{F},
$$

where $\beta$ is a normalizing array of $1 \times n$ dimension, which indicates the importance or weight assigned to each of the factors considered, while $F$ is an array of $n \times 1$ dimension, whose elements are either one or zero, representing whether each factor is satisfactory or unsatisfactory, respectively. The values of $A_{c}$ lie in the range [0,1]. The matrix product can be expressed as a sum, given in (2) as:

$$
A_{c}=\sum_{i=1}^{n} \boldsymbol{\beta}_{i} \times \boldsymbol{F}_{i}
$$

Where $i$ is an index representing the number of factors considered, ranging from 1 to $n ; \beta_{i}$ is the importance weight for the $i^{\text {th }}$ factor; and $F_{i}$ represents whether the requirement for the $i^{\text {th }}$ factor is satisfied or not. The sum of the weights must be equal to one, as given in (3):

$$
\sum_{i=1}^{n} \beta_{i}=1 \text {. }
$$

The criterion is formulated to provide both robustness and flexibility, allowing adjustment to the factors which are considered important, depending on the use case and context. Fig. 2 shows an illustration of the entire AIenabled PHM process, from design and algorithm development using standards all the way to the application 
of the acceptability criterion and then to subsequent certification, implementation, and continuous monitoring.

\section{DEMONSTRATION AND DISCUSSION}

\section{A. Typical Application of Acceptability Criterion}

The use of the acceptability criterion is succinctly demonstrated in this section. It requires a list of all the factors that need to be satisfied to assure regulators that due diligence has been carried out. As stated earlier, such a list of factors and the corresponding importance weighting would typically be context-specific. A team of engineers from regulatory agencies, certification bodies and the asset team should determine the suitable importance weighting for each factor. For demonstration purposes, Table II shows a list of factors and the importance weighting assigned to each of them for a given AI-enabled PHM solution.

From the somewhat arbitrary assignments in Table II, the acceptability criterion is computed using the formula in (2) to obtain $A_{c}=0.75$. A suitable acceptance threshold is then set by the certification body, say $A_{c} \geq 0.9$, depending on how safety-critical the monitored system or unit is. To achieve certification, the value of $A_{c}$ must be increased by at least satisfying any two of cyber-security, explainability, and having a legal and ethical policy, which will raise the $A_{c}$ score to $\geq 0.9$. This demonstration shows how flexibly the acceptability criterion can be applied and contextualized. Furthermore, its robustness property stems from its amenability to different levels of scrutiny, which may be very high level, or very detailed, depending on industry-specific requirements.

TABLE II.

APPLICATION OF THE ACCEPTABILITY CRITERION

\begin{tabular}{|c|l|c|c|c|c|}
\hline$i$ & \multicolumn{1}{|c|}{ Factor } & Satisfied $?$ & $\boldsymbol{F}$ & Weight, $\boldsymbol{\beta}$ & $\boldsymbol{\beta} \boldsymbol{F}$ \\
\hline 1 & Safety & Yes & 1 & 0.20 & 0.20 \\
\hline 2 & Reliability & Yes & 1 & 0.10 & 0.10 \\
\hline 3 & Cyber-security & No & 0 & 0.10 & 0.00 \\
\hline 4 & Explainability & No & 0 & 0.10 & 0.00 \\
\hline 5 & Interpreatibility & Yes & 1 & 0.05 & 0.05 \\
\hline 6 & Accurate preditions & Yes & 1 & 0.20 & 0.20 \\
\hline 7 & $\begin{array}{l}\text { Follows sector-specific } \\
\text { standards }\end{array}$ & Yes & 1 & 0.10 & 0.10 \\
\hline 8 & Legal and ethical policy & No & 0 & 0.05 & 0.00 \\
\hline 9 & $\begin{array}{l}\text { Third-party testing, } \\
\text { verification and validation }\end{array}$ & Yes & 1 & 0.10 & 0.10 \\
\hline
\end{tabular}

\section{B. Other Considerations}

a) Hardware considerations: sensors selection and placement affect the quality of data and condition monitoring capabilities. Optimal sensor placement methodologies must be explored and developed, especially when migrating existing or legacy systems to AI- enabled PHM. Also, interoperability across different OEM modules and data storage equipment should follow recognized standards.

b) Software considerations: troubleshooting and debugging spurious predictions or software faults for blackbox models is potentially tricky. This relates directly to explainability of AI. All the core tenets on XAI, some of which were discussed earlier, along with software engineering best practices can help in this regard.

c) Legacy assets and convergence issues: a possible solution that promises to provide a bridge for integration of new processes or solutions with existing ones is the concept

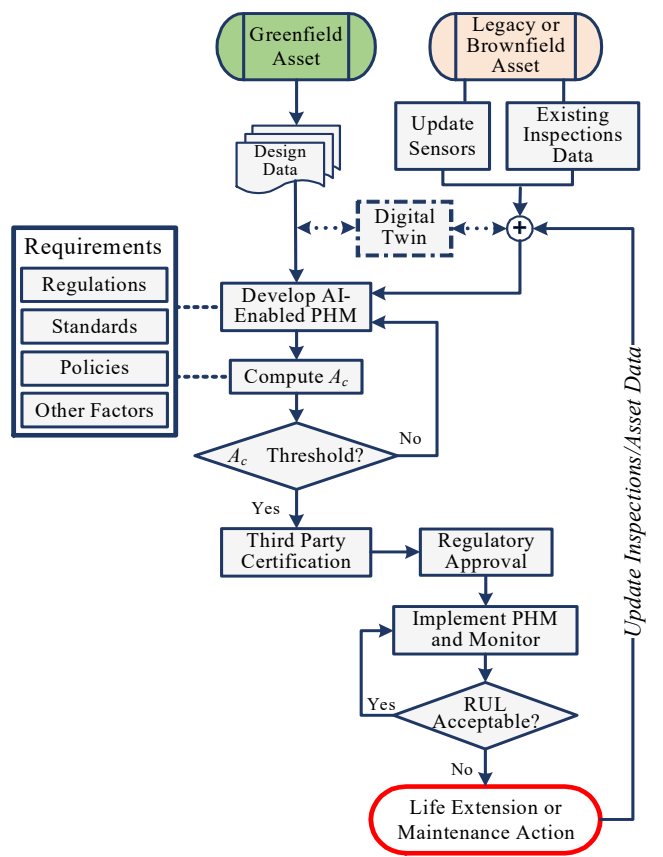

Figure 2. Overall flow of AI-enabled PHM process within the context of compliance with standards and regulations.

of digital twins. Again, new technologies or concepts automatically trigger corresponding regulation and compliance issues. Digital twin technologies, which implicitly incorporate AI-enabled PHM, must also be qualified and certified for use [30]. For organization-wide deployment, the relevant change management issues to be addressed include upgrade of sensors, data management and documentation, personnel training and competency development, human factors, upgrade of user interfaces, and scalability across the entire asset portfolio.

\section{Potential Challenges}

Cyber-physical systems raise additional security challenges, which increases cost and complexity, and introduce additional compliance requirements, thereby raising the barrier to adoption. Also, legal and liability issues add another layer of challenges which should be carefully legislated to encourage innovation.

Integration of legacy facilities and the convergence of old systems with new ones, both in terms of hardware and software, presents additional personnel and competency development requirements. Human factors issues must be addressed such that user-interfaces and system troubleshooting modules are easily comprehensible. Personnel training should incorporate the core principles of explainability and interpretability so that operators and asset managers can draw the full benefits of the decision support capabilities that AI-enabled PHM provides.

As a final yet important point, to avoid an overload of standards and the potential confusion that it can trigger, professional societies should coordinate standards development, addressing the various stages of AI-enabled PHM specific to different fields. The SAE's work in this regard, with different committees addressing sectorspecific PHM standards, is a good model to follow. 


\section{CONCLUSION}

There is no doubt that the recent rapid increase in the application of AI in engineering systems is bound to continue. Consequently, professionals as well as regulators must find creative ways of establishing a productive nesting ground for the successful maturation of AI-enabled technologies, one of which is data-driven prognostics. Professional organizations like the IEEE, ISO, SAE, and other organizations like MIMOSA, have indeed laid the foundation in terms of defining architectures and developing some associated standards. Formulation of ancillary regulations, however, lag standards development. This study proposed a flexible yet robust way of approaching certification and regulation of AI-enabled PHM, by the utilization of a user-definable acceptability criterion. The application of the acceptability criterion was demonstrated in this paper, and if fully exploited, will help serve as a basis for establishing regulatory sandboxes, which are necessary at this stage of technological readiness of AI-enabled PHM. Ultimately, this should be one amongst the many small leaps that must be made towards the actualization of a fully functional regulatory framework for AI-enabled PHM.

\section{REFERENCES}

[1] S. Ochella and M. Shafiee, "Artificial intelligence in prognostic maintenance," Proc. 29th Eur. Saf. Reliab. Conf. ESREL 2019, pp. 3424-3431.

[2] J. Bird and G. Shao, "A view of standards for prognostics and health management," Int. J. Progn. Heal. Manag., vol. 4, no. 2, p. 7, 2013.

[3] Y. Zhou, J. Bo, and T. Wei, "A review of current prognostics and health management system related standards," Chem. Eng. Trans., vol. 33, pp. 277-282, 2013.

[4] G. W. Vogl, B. A. Weiss, and M. A. Donmez, "Standards for prognostics and health management (PHM) techniques within manufacturing operations," PHM 2014 - Proc. Annu. Conf. Progn. Heal. Manag. Soc. 2014, pp. 576-588.

[5] J. W. Sheppard and J. D. Debruycker, "An investigation of current and emerging standards to support a framework for prognostics and health management in automatic test systems," 2018 IEEE AUTOTESTCON, National Harbor, MD, USA, pp. 1-7, Nov. 2018.

[6] S. Chang, L. Gao, and Y. Wang, "A review of Integrated Vehicle Health Management and prognostics and health management standards," 2018 International Conference on Sensing, Diagnostics, Prognostics, and Control, (SDPC) Xi'an, China, August 2018, pp. 476-481.

[7] L. NíFhaoláin, A. Hines, and V. Nallur, "Assessing the appetite for trustworthiness and the regulation of artificial intelligence in europe," CEUR Workshop Proc., vol. 2771, pp. 133-144, 2020.

[8] International Organization for Standardization, ISO 133811:2015 Condition monitoring and diagnostics of machines - Prognostics-Part 1: General guidelines. 2015, pp. 1-21.

[9] J. W. Sheppard, M. A. Kaufman, and T. J. Wilmer, "IEEE standards for prognostics and health management," IEEE Aerosp. Electron. Syst. Mag., vol. 24, no. 9, pp. 34-41, 2009.

[10] P. W. Kalgren, C. S. Byington, M. J. Roemer, and M. J. Watson, "Defining PHM, a lexical evolution of maintenance and logistics," 2006 IEEE AUTOTESTCON, Anaheim, CA, USA, pp. 353-358.

[11] MIMOSA, "MIMOSA Open System Architecture for Condition-Based Maintenance," OSA-CBM Version 3.3.1,
2010. https://www.mimosa.org/specifications/osa-cbm-3-3$1 /$.

[12] MIMIOSA, "MIMOSA Open System Architecture for Enterprise Application Integration," OSA-EAI Version 3.2.3a, 2014. https://www.mimosa.org/specifications/osaeai-3-2-3a/.

[13] S. Ochella, M. Shafiee, and C. Sansom, "Adopting machine learning and condition monitoring P-F curves in determining and prioritizing high-value assets for life extension," Expert Syst. Appl., vol. 176, p. 114897, 2021.

[14] G. W. Vogl, B. A. Weiss, and M. A. Donmez, "Standards related to prognostics and health management (PHM) for manufacturing," US Department of Commerce, National Institute of Standards and Technology, 2014.

[15] ISO/IEC/IEEE, "ISO/IEC/IEEE International Standard Systems and software engineering — Life cycle processes - Risk management," ISO/IEC/IEEE 16085:2021(E). pp. $1-60,2021$.

[16] J. G. P. Lisboa, "Industrial use of safety-related artificial neural networks," HSE Contract Research Report 327/2001. HMSO, pp. 1-36, 2001.

[17] S. Eldevik, C. Agrell, A. Hafver, and F. B. Pedersen, "AI + Safety: Safety implications for artificial intelligence," DNV-GL Group Technology and Research Position Paper 2018.

[18] IEEE, "IEEE draft standard for model process for addressing ethical concerns during system design," IEEE P7000/D5, February 2021, pp. 1-79, 2021.

[19] European Commission, "Report on the safety and liability implications of artificial intelligence, the internet of things and robotics," 2020.

[20] S. Anastasi, M. Madonna, and L. Monica, "Implications of embedded artificial intelligence - Machine learning on safety of machinery," Procedia Comput. Sci., vol. 180, pp. 338-343, 2021.

[21] DNV-GL, "Trustworthy industrial AI systems," DNV-GL Group Technology and Research, Position Paper 2019. pp. $1-40,2019$.

[22] International Organization for Standardization/ International Electrotechnical Commission, ISO/IEC TR 24028:2020 Information technology - Artificial Intelligence - Overview of trustworthiness in artificial intelligence. 2020.

[23] B. Shneiderman, "Bridging the gap between ethics and practice: guidelines for reliable, safe, and trustworthy human-centered AI slystems," ACM Trans. Interact. Intell. Syst., vol. 10, no. 4, p. 26, 2020.

[24] T. Hinrichs and B. Buth, "Can AI-based components be part of dependable systems?," 2020 IEEE Intell. Veh. Symp.,pp. 226-231, 2020.

[25] DNV-GL, "DNVGL-RP-0510 Framework for assurance of data-driven algorithms and models," April, 2020.

[26] D. Leslie, "Understanding artificial intelligence ethics and safety: a guide for the responsible design and implementation of AI systems in the public sector," Alan Turing Inst., Jun. 2019.

[27] P. Laplante, D. Milojicic, S. Serebryakov, and D. Bennett, "Artificial intelligence and critical systems: from hype to reality," Computer (Long. Beach. Calif)., vol. 53, no. 11, pp. 45-52, Nov. 2020.

[28] K. Goebel and R. Rajamani, "Policy, regulations and standards in prognostics and health management," Int. J. Progn. Heal. Manag., vol. 12, no. 1, 2021.

[29] C. Agrell, S. Eldevik, A. Hafver, F. B. Pedersen, E. Stensrud, and A. Huseby, "Pitfalls of machine learning for tail events in high risk environments," Saf. Reliab. - Safe Soc. a Chang. World - Proc. 28th Int. Eur. Saf. Reliab. Conf. ESREL 2018, pp. 3043-3052, 2018.

[30] DNV-GL, "DNVGL-RP-A204 Qualification and assurance of digital twins," vol. October, 2020. 